\title{
RESILIENCIA EN EDIFICACIONES CON AISLADORES SÍSMICOS
}

\section{RESILIENCE IN BUILDINGS WITH SEISMICISOLATORS}

\author{
Frances V. Benites Jessen ${ }^{1}$ \\ Esther J. Vargas Chang ${ }^{2}$
}

\author{
RECEPCIÓN: 18 DE OCTUBRE DE 2021 \\ ACEPTACIÓN: 10 DE NOVIEMBRE DE 2021
}

\section{RESUMEN}

En los últimos años se ha incrementado la búsqueda de aumentar la resiliencia en las edificaciones con la finalidad de brindar mayor seguridad y sostenibilidad a las ciudades. La presente investigación empleó una metodología descriptivo- comparativa basada en la recopilación de datos de artículos científicos que recogen las respuestas sísmicas de edificaciones convencionales y de edificaciones que cuentan con aisladores sísmicos. A partir de esto se compararon los resultados obtenidos ante sismos acontecidos y las consecuencias que surgen al no implementar estos dispositivos. Dentro del análisis del desempeño sísmico, se evaluaron y compararon las respuestas en aceleraciones y desplazamientos de edificaciones aisladas y convencionales. Se logró demostrar que los aisladores sísmicos reducen las aceleraciones y desplazamientos, debido a la independización que se produce entre la base y la superestructura. Finalmente, se resumen los resultados de la comparación del desempeño sísmico y el análisis de consecuencias que se producen al no implementar estos dispositivos. Por lo tanto, el sistema aislado aporta una eficiencia dentro del desempeño sísmico disminuyendo o evitando daños estructurales en edificaciones, pérdidas de vidas y tiempos de inhabitabilidad ante un sismo, que a su vez contribuyen con el incremento de la resiliencia y aminoran la vulnerabilidad de las edificaciones.

Palabras clave: Aisladores sísmicos, eficiencia, inhabitabilidad, resiliencia, desempeño sísmico, sustentable.

\begin{abstract}
In recent years, the search to increase resilience in buildings has increased in order to provide greater safety and sustainability to cities. The present research used a descriptive-comparative methodology based on the collection of data from scientific articles that collect the seismic responses of conventional buildings and buildings that have isolated seismics. From this, the results obtained in the event of earthquakes and the consequences that arise from not implementing these devices were compared. Within the seismic performance analysis, the responses in acceleration and displacement of isolated and conventional buildings were evaluated and compared. It will be shown that seismic isolators reduce accelerations and displacements, due to the independence that occurs between the base and the superstructure. Finally, the results of the comparison of the seismic performance and the analysis of consequences produced by not implementing these devices are summarized. Therefore, the isolated system provides an efficiency within the seismic performance reducing or avoiding structural damage in buildings, loss of life and habitation times in the event of an earthquake, which in turn increases resilience and reduces the vulnerability of buildings.
\end{abstract}

Keywords: Seismic isolators, efficiency, uninhabitable, resilience, seismic performance, sustainable.

1 Universidad Ricardo Palma. Facultad de Ingeniería. <frances.benites@urp.edu.pe>

2 Universidad Ricardo Palma. Facultad de Ingeniería. <esther.vargas@urp.edu.pe $\geq$ 


\section{INTRODUCCIÓN}

A lo largo de los años, se ha observado las devastadoras consecuencias que se producen después de un sismo, como pérdidas de vida, daños estructurales y tiempos de inhabitabilidad.

El Perú se encuentra ubicado en una zona altamente sísmica, en el borde oriental dentro del Círculo de Fuego del Pacífico. Nuestra normativa de Diseńo Sismorresistente contempla diseñar edificaciones capaces de resistir sismos de escasa intensidad o que, en su defecto, produzcan daños mínimos. Sin embargo, esto no asegura la operacionalidad de la edificación, ni se prevé anular tiempos de inhabitabilidad después de un sismo. Esto último recae en la importancia de generar una edificación que reúna esas características con el fin de convertirse en una estructura resiliente.

Por ello, el presente artículo se centra en los resultados de investigaciones consultadas acerca del desempeño sísmico de edificaciones que han contado con aislamiento sísmico y cómo este ha optimizado su comportamiento y mermado las consecuencias de dańos estructurales lo que ha influido en la nulidad de los tiempos de inhabitabilidad. De tal manera, denota un comportamiento resiliente.

La investigación presenta los resultados de las respuestas sísmicas ante el sismo de Northridge, Los Ángeles, en 1994, de dos hospitales, el University of Southern California (USC), el cual dispone de aislamiento sísmico, y el Olive View Hospital (OVH), el cual no dispone de aislamiento sísmico. Así mismo, los resultados de las respuestas sísmicas ante el sismo de Maule, Chile, en 2010, del edificio Comunidad de Andalucía, conformado por dos torres gemelas, una de las cuales se encuentra aislada sísmicamente mientras que la otra mantiene la estructura convencional.

\section{ANTECEDENTES Y CONTEXTO EN LA ACTUALIDAD EN EL PERÚ}

\subsection{Antecedentes}

La búsqueda por aumentar la resiliencia en edificaciones se remonta a tiempos pasados, en los cuales el ingenio humano ha intentado sobreponerse al impacto de grandes desastres como los sismos. El Perú no ha sido ajeno a ello, lo que se refleja en la Cultura Caral, que es considerada la civilización más antigua de América.

Caral está ubicada, según el Reglamento Nacional de Edificaciones E.030 Diseño sismorresistente, dentro de la zonificación 4. Se considera, por eso, que su ubicación es propensa a percibir movimientos telúricos de mayor intensidad. Es por ello que desde aquel tiempo esta civilización aplicaba técnicas para "aislar" sísmicamente las estructuras que construían.

La técnica era aislar la construcción del terreno mediante colocación de bolsas tejidas denominadas "Shicras". Según Asencios [1], estos sacos o bolsas están compuestas de tejidos de mallas con soguillas a las cuales se le rellenaban de piedras. Por otro lado, Iwaki, Rubiños y Vargas [2] enfatizan que mediante este sistema de construcción se mermó la energía sísmica y permitió conseguir estabilizar las construcciones piramidales características de la cultura. De esta manera, esta técnica ha permitido que estas construcciones piramidales, actualmente, permanezcan dentro de un estado óptimo.

\subsection{Contexto en la actualidad peruana}

En la actualidad, el Perú cuenta con una normativa que respalda la inclusión de aisladores sísmicos en edificaciones debido a la ubicación del país. En el año 2019 se incorporó la Norma Técnica 
E.031 Aislamiento Sísmico, que exige la aplicación obligatoria de este sistema dentro de edificaciones de categoría "A1"; es decir, en edificaciones esenciales como establecimientos de salud ubicadas en zonas sísmicas 3 y 4 . Sin embargo, edificaciones de otra categoría no están dentro de este régimen obligatorio.

Por otro lado, Talavera [3], en su investigación "Experiencia nacional en edificaciones con sistemas de protección sísmica”, indica que el Perú cuenta con edificaciones de distinta categoría que ya han empleado este sistema de protección sísmica. Dentro de esta investigación se resaltan 19 edificaciones aisladas en un estado de "culminado", 4 en proceso de construcción y 16 en etapa de proyecto.

Debido a que aún no se tiene una data de comportamiento post-sismo de estas edificaciones para poder realizar el análisis respectivo, esta investigación tiene como foco principal dar a conocer los resultados del desempeño sísmico en edificaciones que han apostado por este sistema de protección sísmica. De esta manera, se podrá conocer y visualizar los resultados ante eventos sísmicos acontecidos.

\section{METODOLOGÍA}

Según la definición de Hernández, Fernández y Baptista [4], la metodología aplicada en el presente artículo se caracteriza por ser de carácter descriptivo-comparativo, porque se describieron los resultados del desempeño sísmico de las edificaciones tomadas en cuenta en esta investigación ante distintos eventos sísmicos. A partir de este procedimiento, se compararon las respuestas sísmicas y se analizaron las consecuencias que surgen al no incluir este sistema antisísmico.

La herramienta computacional utilizada fue el programa Excel, el cual nos permitió realizar el cálculo comparativo porcentual de variaciones con respecto a las aceleraciones y desplazamientos con la finalidad de plasmarlos de forma dinámica a través de gráficos de barras y circulares.

\section{RESULTADOS}

Comparación del desempeño sísmico entre el USC Hospital y el Olive View Hospital tras el sismo de Northridge, Los Ángeles en 1994:

TABLA 1. Variación porcentual (\%Var) de las aceleraciones registradas en dirección " $x$ " entre el USC Hospital y el Olive View Hospital frente al sismo de Northridge, Los Ángeles, en 1994.

\begin{tabular}{cccc}
\hline \multicolumn{4}{c}{ Aceleración $(\mathrm{g})$} \\
\hline & $\begin{array}{c}\text { Hospital aislado } \\
\text { USC Hospital }\end{array}$ & Hospital no aislado & \\
Olive View Hospital & \\
Nivel & Dirección "x" & Dirección "x" & $\%$ Var \\
Techo & 0.21 & 2.31 & $90.91 \%$ \\
Planta baja & 0.13 & 0.82 & $84.15 \%$ \\
Terreno & 0.49 & 0.91 & $46.15 \%$ \\
\hline
\end{tabular}

Fuente: Elaboración propia

Datos: Nagarajaiah \& Xiahong [5) y Celebi [6] 
TABLA 2. Variación porcentual (\%Var) de las aceleraciones registradas en dirección "y" entre el USC Hospital y el Olive View Hospital frente al sismo de Northridge, Los Ángeles, en 1994.

\begin{tabular}{cccc}
\hline \multicolumn{3}{c}{ Aceleración $(\mathrm{g})$} \\
\hline & $\begin{array}{c}\text { Hospital aislado } \\
\text { USC Hospital }\end{array}$ & $\begin{array}{c}\text { Hospital no aislado } \\
\text { Olive View Hospital }\end{array}$ & \\
Nivel & Dirección “y” & Dirección “y” & $\%$ Var \\
Techo & 0.19 & 0.79 & $75.95 \%$ \\
Planta baja & 0.14 & 0.42 & $66.67 \%$ \\
Terreno & 0.22 & 0.61 & $63.93 \%$ \\
\hline
\end{tabular}

Fuente: Elaboración propia

Datos: Nagarajaiah \& Xiahong [5] y Celebi [6]

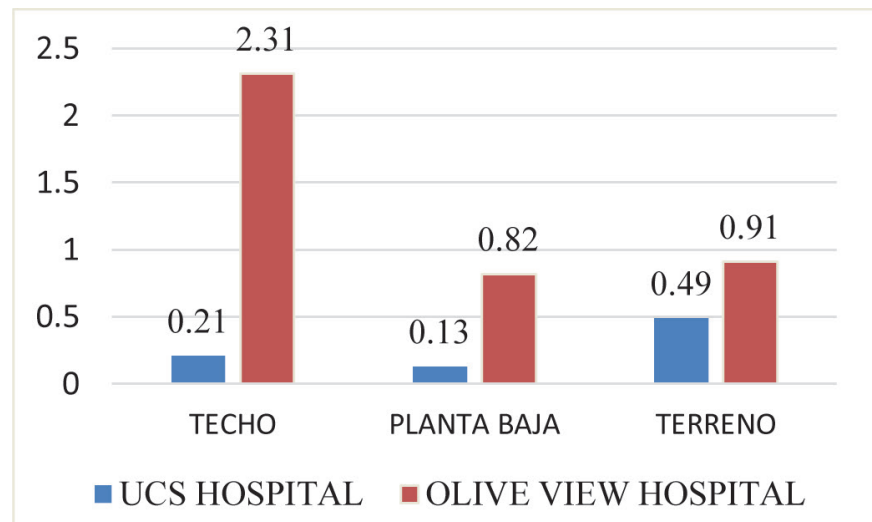

Figura 1. Comparativa de las aceleraciones en dirección " $x$ ” entre el USC Hospital y el Olive View frente al sismo de Northridge, Los Ángeles, en 1994. Fuente: Elaboración propia

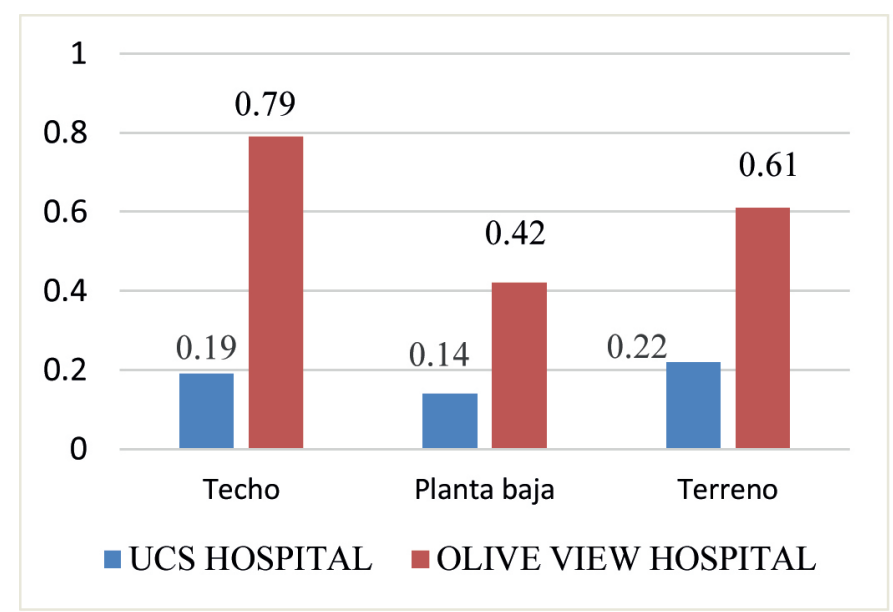

Figura 2. Comparativa de las aceleraciones en dirección "y" entre el USC Hospital y el Olive View frente al sismo de Northridge, Los Ángeles, en 1994. Fuente: Elaboración propia 
TABLa 3. Variación porcentual (\%Var) de los desplazamientos registrados en dirección " $x$ " entre el USC Hospital y el Olive View Hospital frente al sismo de Northridge, Los Ángeles, en 1994.

\section{Desplazamientos $(\mathrm{cm})$}

\begin{tabular}{cccc}
\hline & $\begin{array}{c}\text { Hospital aislado } \\
\text { USC Hospital }\end{array}$ & $\begin{array}{c}\text { Hospital no aislado } \\
\text { Olive New Hospital }\end{array}$ & \\
Nivel & Dirección "x" & Dirección "x" & $\%$ Var \\
Techo & 3.9 & 34.1 & $88.56 \%$ \\
Planta baja & 2.8 & 28.3 & $90.11 \%$ \\
Terreno & 2.3 & 32.6 & $92.94 \%$ \\
\hline
\end{tabular}

Fuente: Elaboración propia

Datos: Nagarajaiah \& Xiahong [5] y Celebi [6]

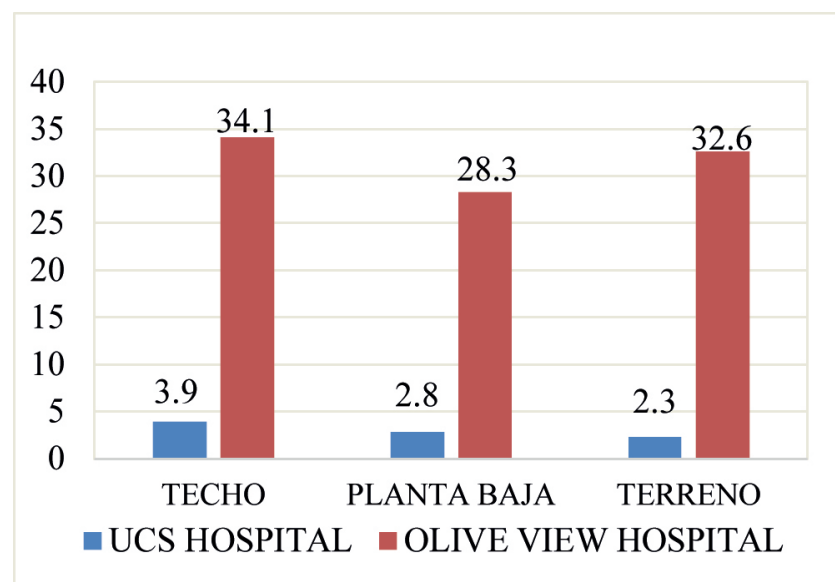

Figura 3. Comparativa de los desplazamientos en dirección " $x$ " entre el USC Hospital y el Olive View Hospital frente al sismo de Northridge, Los Ángeles, en 1994. Fuente: Elaboración propia

TABLA 4. Variación porcentual (\%Var) de los desplazamientos registrados en dirección "y" entre el USC Hospital y el Olive View Hospital frente al sismo de Northridge, Los Ángeles, en 1994.

\begin{tabular}{cccc}
\hline \multicolumn{4}{c}{ Desplazamientos $(\mathbf{c m})$} \\
\hline & Hospital aislado & Hospital no aislado & \\
USC Hospital & Olive New Hospital & \\
Nivel & Dirección “y” & Dirección “y” & $\%$ Var \\
Techo & 5.1 & 19.2 & $73.44 \%$ \\
Planta baja & 3 & 18.4 & $83.70 \%$ \\
Terreno & 2.5 & 15.2 & $83.55 \%$ \\
\hline
\end{tabular}

Fuente: Elaboración propia

Datos: Nagarajaiah \& Xiahong [5] y Celebi [6] 
CIVIL

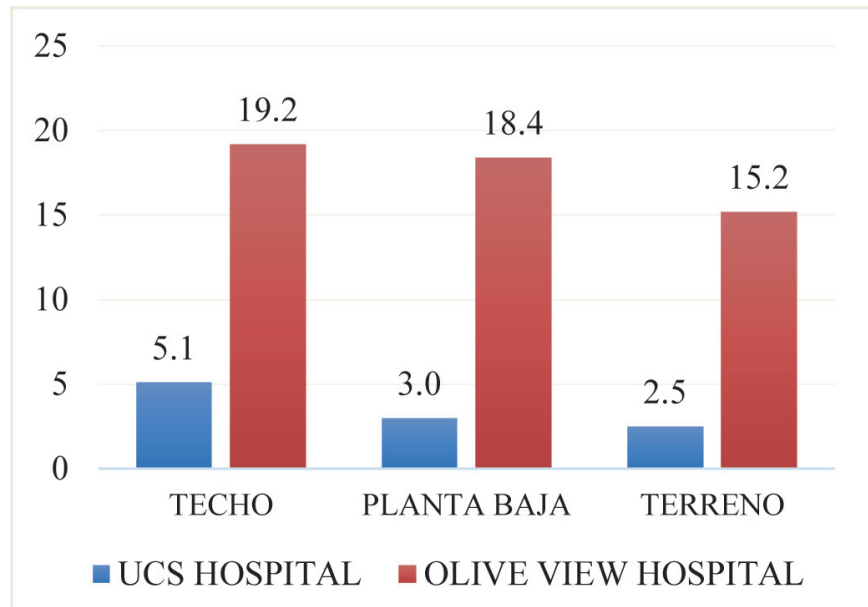

Figura 4. Comparativa de los desplazamientos registrados en dirección “y" entre el USC Hospital y el Olive View Hospital frente al sismo de Northridge, Los Ángeles, en 1994. Fuente: Elaboración propia

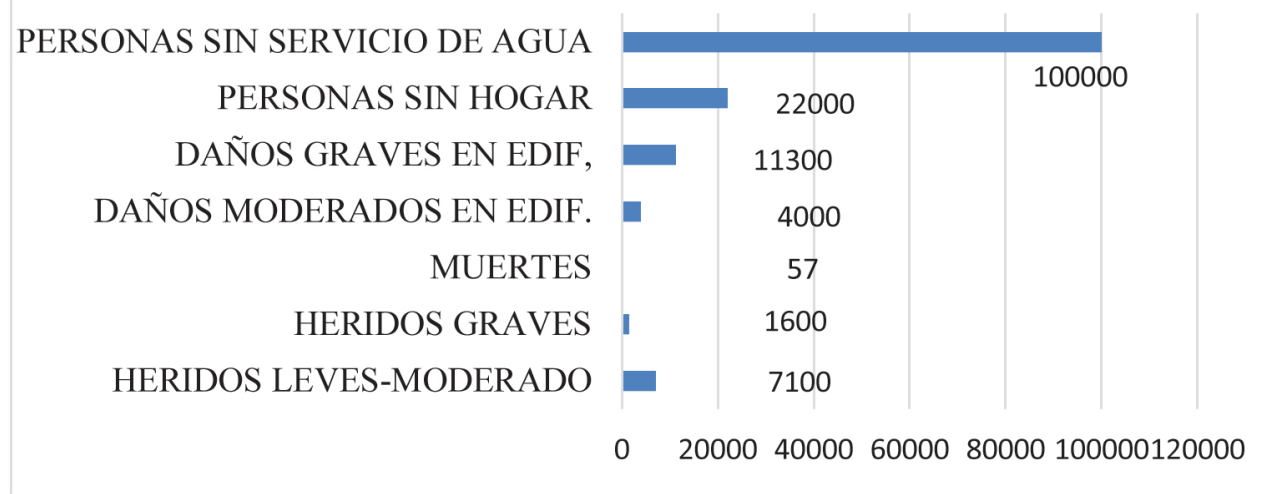

Figura 5. Representación de las consecuencias tras el sismo de Northridge, Los Ángeles, en 1994. Fuente: Elaboración propia Datos: Norton [7] y Boroschek [8]

TABLA 5. Comparativa de funcionalidad entre el USC Hospital y el Olive View Hospital frente al sismo de Northridge, Los Ángeles, en 1994

\begin{tabular}{lcc}
\hline Nivel de funcionalidad & USC Hospital & Olive View Hospital \\
\hline Operacionalidad & $\checkmark$ & X \\
Seguridad & $\checkmark$ & X \\
Estabilidad estructural & $\checkmark$ & X \\
Daños en contenido & X & $\checkmark$ \\
Tiempos de inhabitabilidad & X & X \\
\hline
\end{tabular}

Fuente: Elaboración propia 
Comparación del desempeño sísmico del edificio Andalucía (torre aislada) y de su torre gemela frente al sismo de Maule, Chile, en 2010:

Tabla 6. Variación porcentual (\%Var) de las aceleraciones registradas del edificio Andalucía (torre aislada) en comparación con su torre gemela (torre convencional) frente al sismo de Maule, Chile, en 2010

\begin{tabular}{ccccccccccc}
\hline \multicolumn{10}{c}{ Aceleración (g) } \\
\hline \multicolumn{10}{c}{ Torre Andalucía aislada } & \multicolumn{1}{c}{ Torre Andalucía convencional } \\
\hline NS & EO & VERT & NS & EO & VERT & $\%$ Var & & \\
\hline Tech. & 0,22 & 0,17 & 0,29 & 1,11 & 0,70 & 0,36 & 80,34 & 76,11 & 20,53 \\
\hline
\end{tabular}

Fuente: Elaboración propia

Datos: Asmat \& Taboada [9], Boroschek et al. [10] y Boroschek et al. [11]

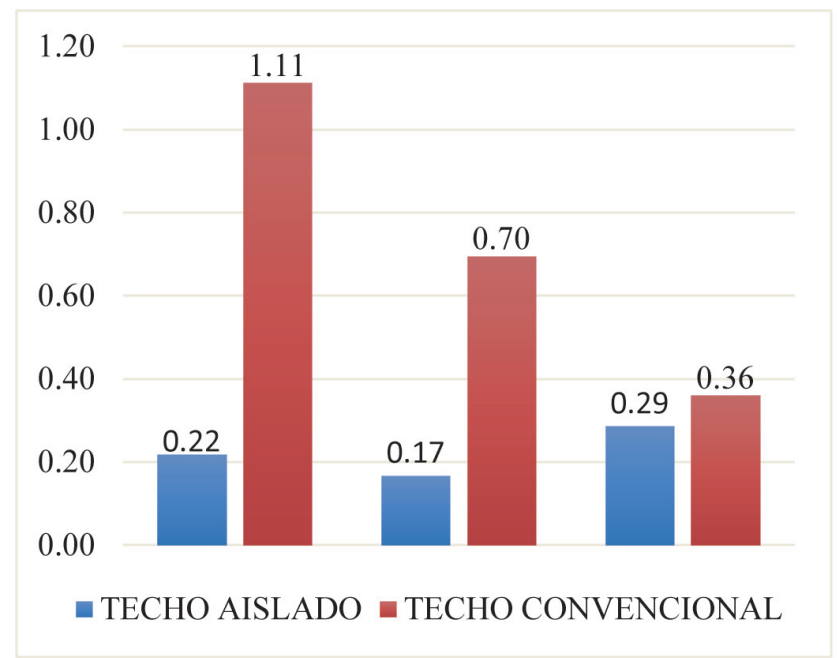

Figura 5. Gráfica comparativa de las aceleraciones entre las torres del edificio Andalucía Fuente: Elaboración propia

Consecuencias registradas tras el sismo de Maule, Chile, en 2010:

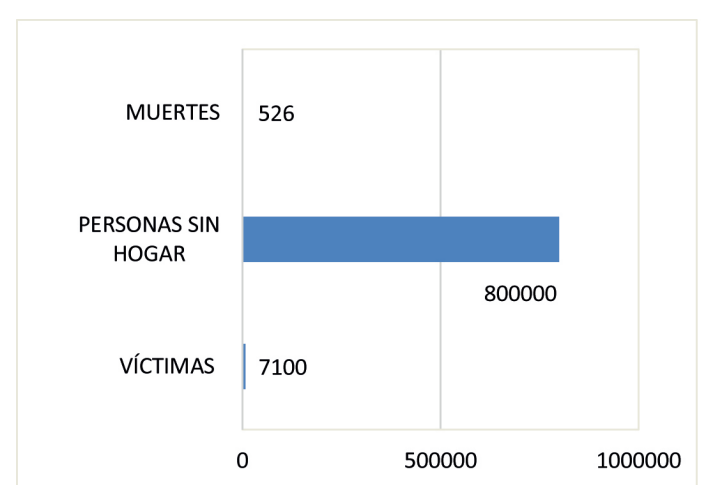

Figura 6. Representación de las consecuencias registradas tras el sismo de Maule, Chile, en 2010. Fuente: Elaboración propia. Datos: De La Llera et al. [12] y Saragoni [13] 


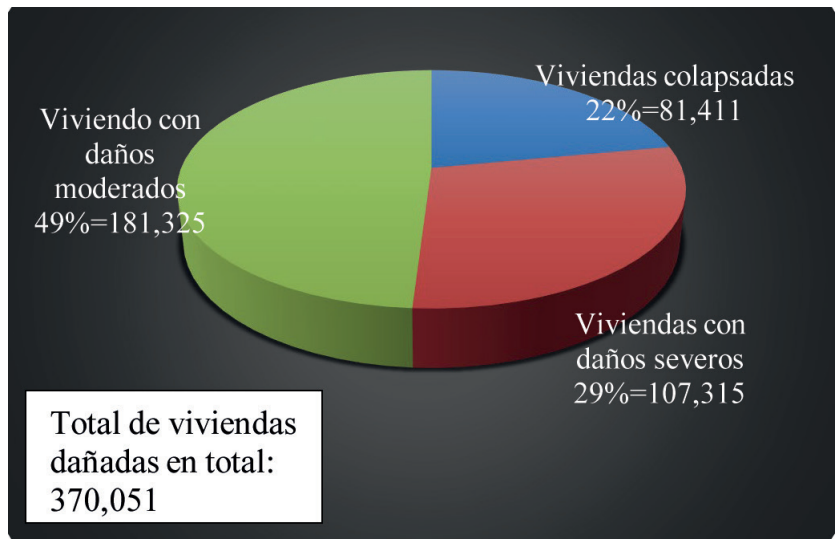

Figura 7. Representación de los niveles de daños presentados en viviendas tras el sismo de Maule, Chile, en 2010.

Fuente: Elaboración propia. Datos: De La Llera et al. [12] y Saragoni [13]

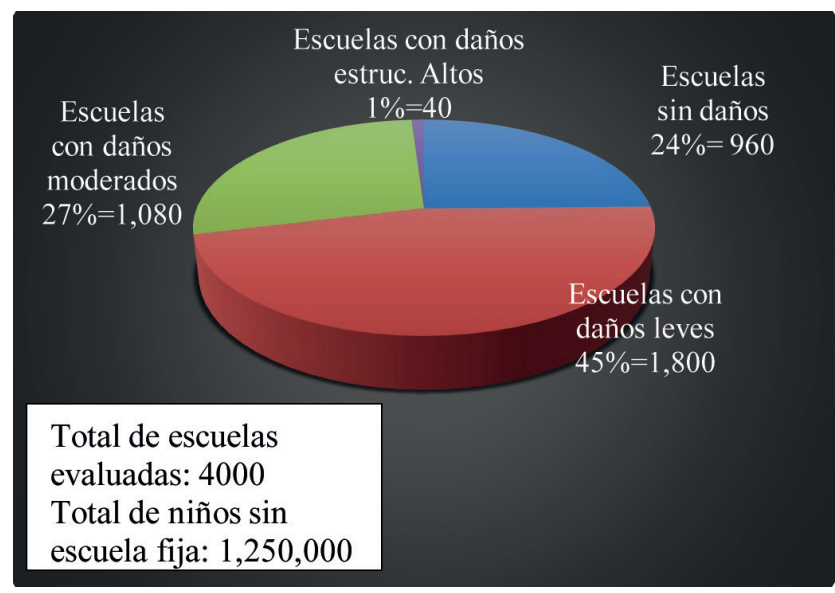

Figura 8. Representación porcentual de daños presentados en las escuelas tras el sismo de Maule, Chile, en 2010.

Fuente: Elaboración propia. Datos: De La Llera et al. [12] y Saragoni [13]

TABLA 7. Comparación de la funcionalidad entre la torre convencional y la torre aislada del edificio Andalucía tras el sismo de Maule, Chile, en 2010.

\begin{tabular}{lcc}
\hline Nivel de funcionalidad & Torre aislada & Torre convencional \\
\hline Operacionalidad & $\checkmark$ & X \\
Seguridad & $\checkmark$ & X \\
Estabilidad estructural & $\checkmark$ & X \\
Daños en contenido & X & $\checkmark$ \\
Tiempos de inhabitabilidad & X & X \\
\hline
\end{tabular}

Fuente: Elaboración propia 


\section{CONCLUSIONES}

Mediante el análisis comparativo del desempeño sísmico, se concluye que el USC Hospital demostró tener una mejor eficiencia y respuesta sísmica que el Olive View Hospital ante el sismo de Northridge, Los Ángeles, en 1994. Así, logró disipar las energías sísmicas captadas y esto se vio reflejado en la reducción de aceleraciones en las direcciones " $\mathrm{x}$ " e "y", sobre todo a nivel del techo. Se apreció en ese sentido que hubo una disminución del $90.91 \%$ y $75.95 \%$, respectivamente. De la misma manera, en los desplazamientos, se produjo la disminución en un $88.56 \%$ y $73.44 \%$ en ambas direcciones. Se concluye que los aisladores sísmicos juegan un papel importante dentro del desempeño sísmico en una edificación, porque disminuyen considerablemente las aceleraciones y desplazamientos, y evitan pérdidas de vidas, daños estructurales o colapsos de edificaciones.

El análisis comparativo del desempeño sísmico de la torre convencional del edificio Comunidad de Andalucía frente a la torre aislada gemela del mismo ante el sismo de Maule, Chile, en 2010, demostró que las aceleraciones de este último fueron disminuidas en un $80.34 \%, 76.11 \%$ y $20.53 \%$ en las direcciones "Ns", "Eo" y "Vertical" respectivamente a nivel del techo, evidenciando la efectividad lograda en el desempeńo sísmico al incluir los aisladores sísmicos en una edificación.

Las consecuencias tras el sismo de Northridge, Los Ángeles, en 1994 fueron devastadores en aquel momento. Se produjeron 7100 heridos leves, 1600 heridos graves, y se sumaron 57 muertes. Se estimaron daños moderados y graves en las edificaciones de 4000 y 11300 , respectivamente. Esto implicó que 22000 personas no tuvieran un hogar fijo por los severos dańos ocurridos en los elementos estructurales y en los contenidos de estas edificaciones. Asimismo, aumentó la paralización de servicios básicos así como del periodo de inhabitabilidad, y se registraron alrededor de 1,000,000 personas sin servicios de agua.

Respecto a la relación resiliencia y aisladores sísmicos, el USC Hospital, el cual contiene aisladores sísmicos, demostró tener un nivel de funcionalidad inmediato según la tabla 5, lo que aseguró la operacionalidad y ocupación inmediata. Además, no hubo reportes de daños estructurales ni daños en sus contenidos. Sin embargo, el Olive View Hospital, el cual no contaba con aislación sísmica, demostró lo contrario. El nivel de funcionalidad inmediata no se produjo por realizarse daños en su contenido. Se concluye de tal manera que los aisladores sísmicos guardan una relación de resiliencia con respecto a una edificación, pues logra que esta pueda continuar operativa a pesar de haberse visto expuesta a una situación compleja, permite una ocupación inmediata, disminuye los tiempos de inhabitabilidad y evita los dańos estructurales y las pérdidas de vidas.

El sismo de Maule, Chile, en 2010, dejó graves consecuencias como la pérdida de vidas de 526 personas, 7100 víctimas y alrededor de 8,000,000 de personas sin un hogar fijo, ya que se registraron cerca de 81411 viviendas colapsadas, 107315 viviendas con dańos severos y 181325 viviendas con daños moderados. A su vez, cerca de 4000 escuelas fueron evaluadas, de las cuales 960 no presentaron daños, 1800 presentaron daños leves, 1080 dańos moderados y 40 presentaron daños estructurales altos, lo que provocó que cerca de 1,250,000 niños permanecieran con actividades escolares suspendidas. Asimismo, mediante la tabla 7, se observa que la torre aislada del edificio Andalucía presentó un nivel funcionalidad inmediata, pues no presentó daños estructurales ni daños en su contenido. Se concluye de tal manera que los aisladores sísmicos permiten que haya una continuación de las actividades básicas después de un sismo de alta intensidad y que no se produzcan dańos severos dentro de la edificación que pongan en peligro la vida de los ocupantes.

Cuando hacemos referencia al término "resiliente" en una edificación, nos referimos a que son capaces de soportar y reponerse a la exposición a acontecimientos de gran impacto como los sismos. La implicancia de la resiliencia en una edificación recae en desarrollar edificaciones sostenibles. Por ello, este concepto se relaciona con los objetivos de desarrollo sostenible (ODS), tales como: 
ODS 3: Asegurar la salud y el bienestar de la sociedad al implementar aisladores sísmicos, ya que garantizan el bienestar de los ocupantes de la edificación y, por consiguiente, la salud de estos.

ODS 4: Asegurar una educación de calidad, puesto que una buena infraestructura educativa conllevaría a una mejora en la calidad educativa. Un ejemplo de ello es el sismo de Maule, en Chile, que dejó a 1,250,000 estudiantes con actividades escolares suspendidas debido a los daños severos que afectaron a las escuelas que no contaban con aisladores sísmicos.

ODS 9: Implementar aisladores sísmicos a una edificación denota una innovación en la industria, pero sobre todo en la infraestructura.

ODS 11: Incluir aisladores sísmicos es parte de la sostenibilidad de una ciudad, ya que reducen el riesgo de colapso en edificaciones ante eventos de desastres naturales, en este caso eventos sísmicos.

\section{REFERENCIAS BIBLIOGRÁFICAS:}

[1] R. Asencios, "Investigaciones de las Shicras en el sitio precerámico de Cerro Lampay", tesis de pregrado, Universidad Nacional Mayor de San Marcos, 2009 [En línea]. Disponible en: https:// cybertesis.unmsm.edu.pe/handle/20.500.12672/3366 [Accedido: 10-ene-2022]

[2] C. Iwaki, A. Rubińos, y J. Vargas, "Sismo Resistencia en las Entrañas de Caral”, 2012 [En línea]. Disponible en: https://silo.tips/download/sismo-resistencia-en-las-entraas-de-caral [Accedido: 10-ene-2022]

[3] A. Talavera, "Experiencia Nacional En Edificaciones Con Sistemas De Protección Sísmica”, en 2017 Gallegos, Casabonne, Arango y Quesada, Ingenieros civiles [En línea]. Disponible en: https:// bit.ly/3HSWqRy [Accedido: 10-ene-2022]

[4] C. Fernández Collado, P. Baptista Lucio, y R. Hernández Sampieri, Metodología de la Investigación. México D. F., México: Editorial McGraw Hill, 2014

[5] S. Nagarajaiah, y S. Xiaohong, "Response of base-isolated Usc hospital building in Northridge earthquake", Journal of structural engineering, vol. 126, no. 10, pp. 1177-1186, 2000. Doi: 10.1061/(ASCE)0733-9445(2000)126:10(1177)

[6] M. Celebi, "Comparison of Northridge Earthquake Response of a Base-Isolated and a conventional Hospital Building”, in 1996 Eleventh World Conference on Earthquake Engineering, pp. 1-8 [En línea]. Disponible en: http://www.iitk.ac.in/nicee/wcee/article/11_25.PDF [Accedido: 10-ene-2022]

[7] J. Norton, A. King, D. Bull, H. Chapman, G. McVerry, T. Larkin, and K. Spring, "Northridge earthquake reconnaissance report", Bulletin of the New Zealand Society for Earthquake Engineering, vol. 27, no. 4, pp. 235-344, 1994 [En línea]. Disponible en: https://doi.org/10.5459/bnzsee.27.4.235-344 [Accedido: 10-ene-2022]

[8] R. Boroschek, O. Moroni, M. Sarrazin, "Analysis of seismic records obtained in isolated structures", in 199812 World Conference on Earthquake Engineering, New Zealand, 2000, pp. 1-8 [En línea]. Disponible en: https:/www.iitk.ac.in/nicee/wcee/article/1122.pdf [Accedido: 10-ene-2022]

[9] C. Asmat, y D. Taboada, "Experiencias del comportamiento de edificios aislados al ser sometidos a terremotos severos", AGER. Ingeniería estructural, 2015. [En línea]. Disponible en: https://bit. ly/34D5FXU [Accedido: 10-ene-2022]

[10] R. Boroschek, R. Retamales, and A. Aguilar, "Seismic response of isolated structures subjected to Mw8.8 Chile earthquake of february 27, 2010", in 2010 The international symposium for CISMID $25^{\text {th }}$ anniversary. Paper no. M-2, pp. 1-9 [En línea]. Disponible en: https://bit.ly/3qcPhFJ [Accedido: 10-ene-2022] 
[11] R. Boroschek, P. Soto, y R. León, "Registros del terremoto del Maule Mw = 8.8", Universidad de Chile, Facultad de Ciencias Físicas y Matemáticas, Informe RENADIC 10/05, 2010 [En línea]. Disponible en: http://www.terremotosuchile.cl/red_archivos/RENAMAULE2010R2.pdf [Accedido: 10-ene-2022]

[12] J. de la Llera et al., "Post- Earthquake Data Collection: The 2010 Maule Collection: The 2010 Maule Earthquake In Chile", in 2014 Tenth U.S National Conference on Earthquake Engineering, Frontiers of Earthquake Engineering, pp. 1-72. [En línea]. Disponible en: https://bit.ly/3JQYYBw [Accedido: 10-ene-2022]

[13] R. Saragoni, "El mega terremoto del Maule de 2010: una lección de buena ingeniería, pero con sorpresas y nuevos desafíos", Revista Anales de la Universidad de Chile, no. 1, pp. 35-56, 2011. Doi: $10.5354 / 0717-8883.2011 .12247$ 